\title{
ZnO Nanowire UV Photodetectors with High Internal Gain
}

\author{
C. Soci, ${ }^{\dagger}$ A. Zhang, ${ }^{\dagger}$ B. Xiang, S. A. Dayeh, D. P. R. Aplin, J. Park, X. Y. Bao, \\ Y. H. Lo, and D. Wang*
}

Department of Electrical and Computer Engineering, Jacobs School of Engineering, University of California, San Diego, 9500 Gilman Drive, La Jolla, California 92093-0407

Received January 15, 2007; Revised Manuscript Received February 26, 2007

\begin{abstract}
ZnO nanowire (NW) visible-blind UV photodetectors with internal photoconductive gain as high as $G \sim 10^{8}$ have been fabricated and characterized. The photoconduction mechanism in these devices has been elucidated by means of time-resolved measurements spanning a wide temporal domain, from $10^{-9}$ to $10^{2} \mathrm{~s}$, revealing the coexistence of fast $(\tau \sim 20 \mathrm{~ns})$ and slow $(\tau \sim 10 \mathrm{~s})$ components of the carrier relaxation dynamics. The extremely high photoconductive gain is attributed to the presence of oxygen-related hole-trap states at the NW surface, which prevents charge-carrier recombination and prolongs the photocarrier lifetime, as evidenced by the sensitivity of the photocurrrent to ambient conditions. Surprisingly, this mechanism appears to be effective even at the shortest time scale investigated of $t<1$ ns. Despite the slow relaxation time, the extremely high internal gain of ZnO NW photodetectors results in gain-bandwidth products (GB) higher than $\sim 10 \mathrm{GHz}$. The high gain and low power consumption of NW photodetectors promise a new generation of phototransistors for applications such as sensing, imaging, and intrachip optical interconnects.
\end{abstract}

Because of its wide band gap $\left(E_{\mathrm{g}}=3.4 \mathrm{eV}\right)$, low cost, and ease of manufacturing, $\mathrm{ZnO}$ is emerging as a potential alternative to $\mathrm{GaN}$ in optoelectronic applications, ${ }^{1}$ including light-emitting diodes, laser diodes, and photodetectors for the UV spectral range. In the past decade, the demonstration of a large variety of functional $\mathrm{ZnO}$ nanowire (NW) devices such as field effect transistors, ${ }^{2,3}$ optically pumped lasers, ${ }^{4,5}$ and chemical and biological sensors ${ }^{6}$ have aroused growing interest in this material. ${ }^{7}$ In particular, $\mathrm{ZnO} \mathrm{NW}$ photodetectors and optical switches have been the subject of extensive investigations. ${ }^{8-18}$

Despite the abundant research on NW photoconduction, ${ }^{19}$ the two main factors contributing to the high photosensitivity of such nanostructures have been scarcely recognized: (1) the large surface-to-volume ratio and the presence of deep level surface trap states in NWs greatly prolongs the photocarrier lifetime; (2) the reduced dimensionality of the active area in NW devices shortens the carrier transit time. Indeed, the combination of long lifetime and short transit time of charge carriers can result in substantial photoconductive gain. ${ }^{20-22}$

In this letter, we present $\mathrm{ZnO} \mathrm{NW}$ photodetectors with large photoresponse; upon UV illumination at relatively low light intensities $\left(I \sim 10 \mu \mathrm{W} / \mathrm{cm}^{2}\right)$, the current in $\mathrm{ZnO}$ NWs increases by several orders of magnitude, which translates

\footnotetext{
* Author to whom the correspondence should be addressed. E-mail: dwang@ece.ucsd.edu.

$\dagger$ These authors contributed equally to this work.
}

to a photoconductive gain of $G>10^{8}$. To elucidate the photoconduction mechanism that involves fast carrier thermalization and trapping at the NW surface and electronhole recombination at extended and localized states, we have studied the photoconductivity of $\mathrm{ZnO}$ NWs by time-resolved measurements and in different ambient conditions (e.g., in air or under vacuum). A physical model was developed to illustrate the origin of the photoconductive gain in semiconductor NWs, where the high density of surface trap states enhances the carrier lifetime. These results demonstrate the uniqueness of NWs for photosensing applications and enable the design of novel photodetector architectures.

The ZnO NWs used in this study were grown by chemical vapor deposition (CVD) using a simple tube furnace at 925 ${ }^{\circ} \mathrm{C}$. The source materials consisted of a mixture of $\mathrm{ZnO}$ powder and graphite (molar ratio of 1:1); $\mathrm{N}_{2}$ was used as the carrier gas. ${ }^{23}$ The as-grown single-crystal ZnO NWs had diameter of $150-300 \mathrm{~nm}$ and length ranging from 10 to $15 \mu \mathrm{m}$. After growth, the NWs were transferred onto a thermally oxidized $\mathrm{Si}$ substrate $\left(600 \mathrm{~nm} \mathrm{SiO}_{2}\right)$, and consequently, Ti/Au (20 nm/160 nm) interdigitated electrodes with $2 \mu \mathrm{m}$ finger spacing were patterned on top of the NWs using optical lithography. A representative SEM image of a single $\mathrm{ZnO} \mathrm{NW}$ device is shown in the inset of Figure 1a.

The results of photocurrent measurements ${ }^{24}$ performed on single NW devices in standard ambient conditions are summarized in Figure 1. Figure 1a shows some typical $I-V$ characteristics of the $\mathrm{ZnO} \mathrm{NWs}$ in dark and under UV 

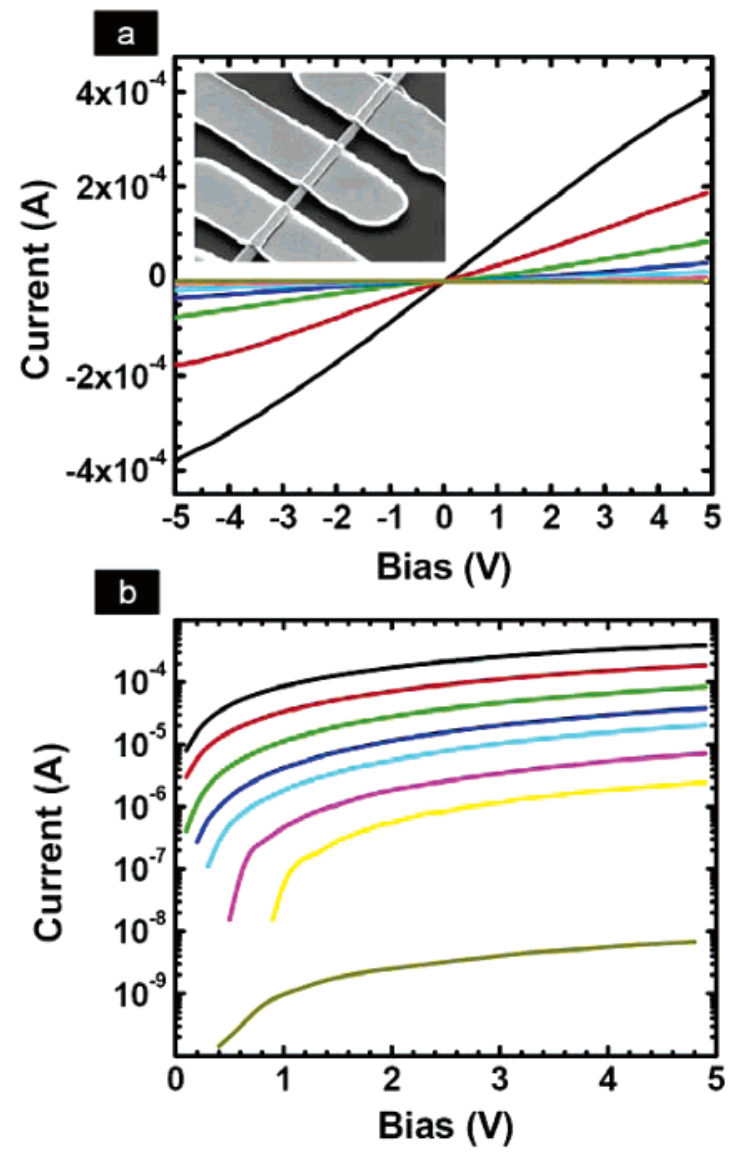

Figure 1. (a) $I-V$ characteristics of a single-NW photodetector as a function of light intensity; from top to bottom, the curves were measured at the following intensities: $4 \times 10^{-2} \mathrm{~W} / \mathrm{cm}^{2}$ (black), 4 $\times 10^{-3} \mathrm{~W} / \mathrm{cm}^{2}(\mathrm{red}), 4 \times 10^{-4} \mathrm{~W} / \mathrm{cm}^{2}$ (green), $1.3 \times 10^{-4} \mathrm{~W} / \mathrm{cm}^{2}$ (blue), $4 \times 10^{-5} \mathrm{~W} / \mathrm{cm}^{2}$ (cyan), $1.3 \times 10^{-5} \mathrm{~W} / \mathrm{cm}^{2}$ (magenta), 6.3 $\times 10^{-6} \mathrm{~W} / \mathrm{cm}^{2}$ (yellow), and in dark (brown). Inset is the SEM image of a typical $\mathrm{ZnO} \mathrm{NW}$ device (obtained at $45^{\circ}$ tilt angle); the spacing between the interdigitated electrodes is $2 \mu \mathrm{m}$. (b) The $I-V$ curves presented in Figure 1a are replotted on a natural logarithmic scale.

illumination $(\lambda=390 \mathrm{~nm})$ at various light intensities; the $I-V$ curves are linear around zero applied bias, which indicates good ohmic behavior of the Ti/Au contacts. ${ }^{7}$ The linear increase of the current with applied bias $(V)$ is due to the increase of the carrier drift velocity, hence the reduction of the transit time $\left(T_{\mathrm{t}}=l^{2} / \mu V\right.$, where $\mu$ is the carrier mobility and $l$ is the separation between the electrodes). The current measured in the NW increases significantly under illumination: by varying the light intensity from $6.3 \mu \mathrm{W} / \mathrm{cm}^{2}$ to 40 $\mathrm{mW} / \mathrm{cm}^{2}$, the current increases from 2 to 5 orders of magnitude, as seen in Figure 1b, where the $I-V$ curves have been redrawn on a natural logarithmic scale.

Because of the high surface-to-volume ratio, trapping at surface states drastically affects the transport and photoconduction properties of NWs. Figure 2 shows a schematic of the photoconduction mechanism in the presence of a high density of hole-trap states at the NW surface; upon illumination with photon energy larger than the semiconductor band gap $\left(E_{\mathrm{g}}\right)$, electron-hole pairs are photogenerated and holes are readily trapped at the surface, leaving behind unpaired electrons, which increase the conductivity under an applied electric field (Figure 2a). Schematics of the NW energy band diagrams in dark and under illumination are displayed in parts $\mathrm{b}$ and $\mathrm{c}$ of Figure 2, respectively, illustrating the charge separation process of photogenerated electrons and holes under the intrinsic NW electric field and the occupation of surface states by photogenerated holes. In $\mathrm{ZnO}$, it has been previously shown that the following trapping mechanism is governing the photoconduction in thin films $\mathrm{s}^{25}$ and NWs: $8,9,13,18,26-28$ in the dark (Figure 2b), oxygen molecules are adsorbed on the oxide surface and capture the free electrons present in the n-type oxide semiconductor $\left[\mathrm{O}_{2}(g)+e^{-} \rightarrow\right.$ $\mathrm{O}_{2}^{-}(a d)$ ], and a low-conductivity depletion layer is formed near the surface. Upon illumination at a photon energy above $E_{\mathrm{g}}$ (Figure 2c), electron-hole pairs are photogenerated [ $h v$ $\rightarrow e^{-}+h^{+}$; ; holes migrate to the surface along the potential slope produced by band bending and discharge the negatively charged adsorbed oxygen ions $\left[h^{+}+\mathrm{O}_{2}^{-}(a d) \rightarrow \mathrm{O}_{2}(g)\right]$, and consequently, oxygen is photodesorbed from the surface. The unpaired electrons are either collected at the anode or recombine with holes generated when oxygen molecules are readsorbed and ionized at the surface. This hole-trapping mechanism through oxygen adsorption and desorption in ZnO NWs augments the high density of trap states usually found in NWs due to the dangling bonds at the surface and thus enhances the NW photoresponse. It is well-known that photoconductors with blocking contacts, i.e., with a Schottky barrier at the metal electrode-semiconductor interface, can exhibit hole-trapping in the reversed-bias junction that shrinks the depletion region and allows tunneling of additional electrons into the photoconductor; if electrons pass multiple times, this mechanism yields photoconductive gain greater than unity. ${ }^{29-32}$ Suppressed recombination of charge carriers has also been reported in PIN diodes with blocking contacts ${ }^{33}$ and type II doping superlattices, ${ }^{34,35}$ where the increase of photocurrent lifetime results in large photoconductive gain. Here we propose a similar mechanism to be effective in NWs, where holes are efficiently trapped at surface states (in $\mathrm{ZnO}$ NWs trapping time is increased by oxygen desorption from the surface) and multiple electron passes through the NW can lead to photoconductive gain.

Figure 3a shows the intensity dependence of the photocurrent (calculated as $I_{\text {light }}-I_{\text {dark }}$ ) measured at an applied bias of $V=5 \mathrm{~V}$. At low light intensities, the photocurrent increases linearly with light intensity, consistent with charge carrier photogeneration efficiency proportional to the absorbed photon flux, while at higher light intensities, it deviates below this linearity. The sublinear dependence of the photocurrent on light intensity can be understood by assuming that, at higher illumination intensities, the number of available hole-traps present at the surface is increasingly reduced, leading to the saturation of the photoresponse. In this case, the density of free carriers $(N)$ in the NW can be expressed as

$$
N=\frac{\eta F}{A L} T_{1}(F), \quad T_{1}(F)=T_{1}^{0} \frac{1}{1+\left(F / F_{\mathrm{o}}\right)^{n}}
$$

where $\eta$ is the charge carrier photogeneration quantum 


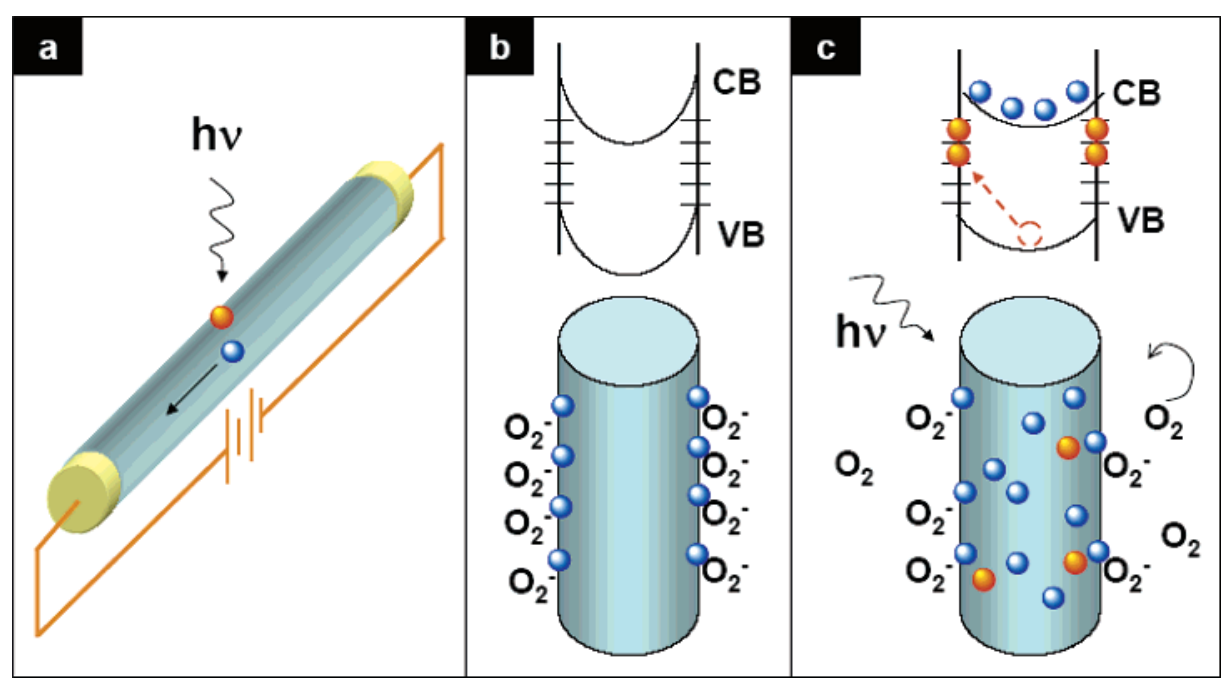

Figure 2. (a) Photoconduction in NW photodetectors. (a) Schematic of a NW photoconductor. Upon illumination with photon energy above $E_{\mathrm{g}}$, electron-hole pairs are generated and holes are readily trapped at the surface. Under an applied electric field, the unpaired electrons are collected at the anode, which leads to the increase in conductivity. (b and c) Trapping and photoconduction mechanism in ZnO NWs: the top drawing in (b) shows the schematic of the energy band diagrams of a NW in dark, indicating band-bending and surface trap states. VB and CB are the valence and conduction band, respectively. The bottom drawing shows oxygen molecules adsorbed at the NW surface that capture the free electron present in the n-type semiconductor forming a low-conductivity depletion layer near the surface. (c) Under UV illumination, photogenerated holes migrate to the surface and are trapped, leaving behind unpaired electrons in the NW that contribute to the photocurrent. In $\mathrm{ZnO} \mathrm{NWs}$, the lifetime of the unpaired electrons is further increased by oxygen molecules desorption from the surface when holes neutralize the oxygen ions.

efficiency, $F$ is the photon absorption rate, $A$ and $L$ are the NW cross section and length, respectively, and $T_{1}$ is the carrier lifetime. As traps are filled, the energy bands flatten and the number of free holes increases; this increases the probability of electron-hole recombination, which is manifested in an absorption-rate-dependent carrier lifetime, $T_{1}(F)$. The analytical expression of $T_{1}(F)$ is given in eq 1 , where $T_{1}^{0}$ is the carrier lifetime at low excitation density, $F_{0}$ is the photon absorption rate when trap saturation occurs, and $n$ is a phenomenological fitting parameter. From the usual expression of the photocurrent $\left(I_{\mathrm{ph}}\right)$, we therefore obtain:

$$
I_{\mathrm{ph}}=q N v A=q \eta\left(\frac{T_{1}^{0}}{T_{\mathrm{t}}}\right) \frac{F}{1+\left(F / F_{\mathrm{o}}\right)^{n}}
$$

where $q$ is the elementary charge and $v=\mu V / l$ is the carrier drift velocity. The solid line in Figure $3 \mathrm{a}$ is the best fit to the data obtained by eq 2 , from which $F_{0}=3.2 \times 10^{6} \mathrm{~s}^{-1}$ and $n=0.7$ have been deduced.

The photoconductive gain is defined as the ratio between the number of electrons collected per unit time and the number of absorbed photons per unit time $\left(G=N_{\mathrm{el}} / N_{\mathrm{ph}}\right),{ }^{36}$ which can be derived from eq 2 by assuming $\eta=1$ for simplicity:

$$
G=\frac{I_{\mathrm{ph}}}{q F}=\left(\frac{T_{1}^{0}}{T_{\mathrm{t}}}\right) \frac{1}{1+\left(F / F_{\mathrm{o}}\right)^{n}}
$$

The first term on the right-hand side of eq 3 is the usual expression for the gain, which is the ratio of carrier lifetime to carrier transit time, and the second term accounts for trap saturation at high excitation intensities. Figure $3 b$ shows the gain derived from the data in Figure 2a according to $G=$ $\left(I_{\mathrm{ph}} / P\right) /(h v / q)$, where $P$ is the power absorbed in the NW. ${ }^{37}$ The extremely long photocarrier lifetime combined with the short carrier transit times due to the reduced dimensionality of the NW devices (i.e., the small spacing between the electrodes) results in photoconductive gain as high as $G=$ $2 \times 10^{8}$; such a gain could allow reaching single-photon detectivity in homogeneous single NWs. ${ }^{38,39}$ The decrease of the gain at relatively high light intensities is a manifestation of hole-trap saturation. The model proposed is in fairly good agreement with the experimental data, where the fitting solid line was plotted from eq 3 , with $F_{0}=3.2 \times 10^{6} \mathrm{~s}^{-1}$ and $n=0.7$. We notice, however, that in addition to trap saturation, the onset of carrier bimolecular recombination at the highest light intensities may also contribute to the shortening of the carrier lifetime. ${ }^{40}$

To determine the charge carrier lifetime, $T_{1}$, we have studied the photocurrent relaxation using time-resolved measurements at low excitation intensity. Figure 4a shows the photocurrent rise upon continuous illumination $(\lambda=390$ $\mathrm{nm}$ ) and the photocurrent decay after removal of incident light at different applied bias. By comparing the curves relative to different biases, it is found that the photocurrent dynamics is independent of the sign and intensity of the external electric field throughout the entire range of applied fields investigated of $|V|<5 \mathrm{~V}$, indicating the absence of space-charge effects. ${ }^{20,21}$ The solid lines represent the best fit to the data obtained by a double-exponential rise and decay functions, from which we deduce a weight-averaged photocurrent rise and decay time constants of $\tau_{\text {rise }}=23 \mathrm{~s}$ and $\tau_{\text {decay }}=33 \mathrm{~s}$. From the conventional expression for the $3 \mathrm{~dB}$ bandwidth of a photodetector, $B=1 / 2 \pi T_{1}$, and the 
a
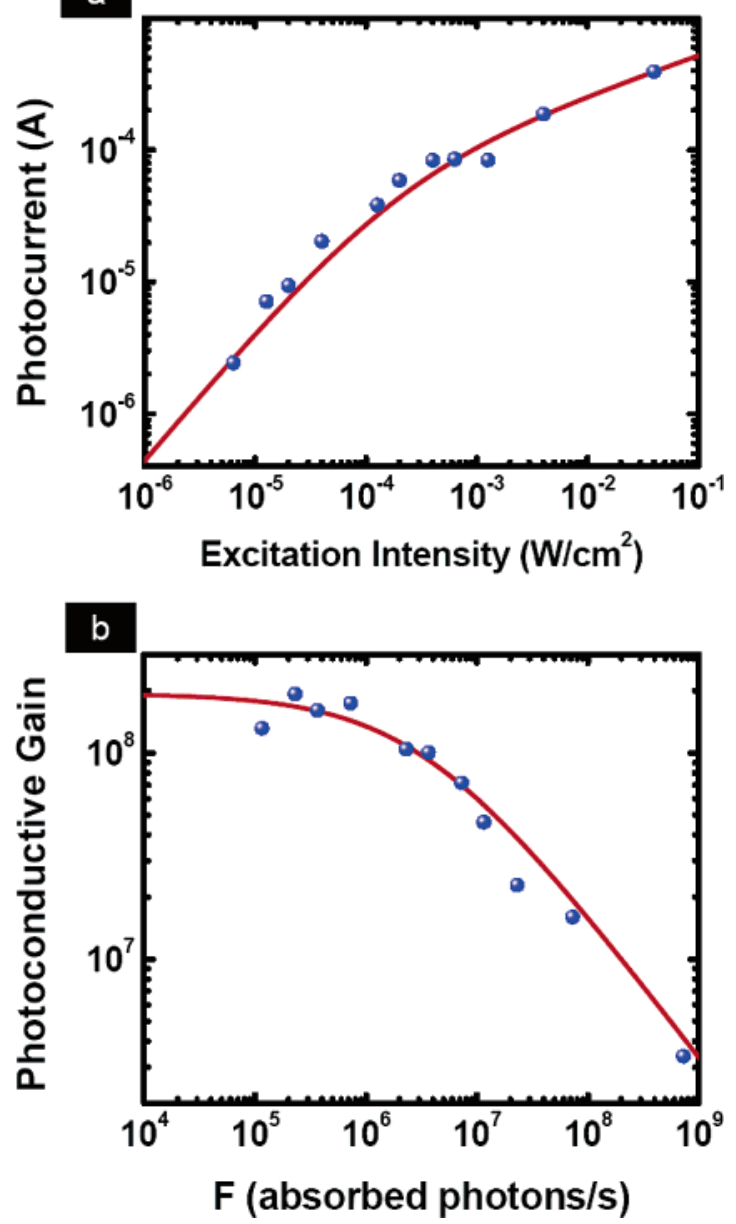

Figure 3. (a) Photocurrent of a single $\mathrm{ZnO} \mathrm{NW}$ device measured as a function of excitation intensity with $5 \mathrm{~V}$ applied bias. (b) Estimate of the photoconductive gain relative to the photon absorption rate in the $\mathrm{ZnO} \mathrm{NW}$.

experimental value of the carrier lifetime $\left(T_{1}=33 \mathrm{~s}\right)$, we obtain for the $\mathrm{ZnO} N W s \quad B \cong 5 \times 10^{-3} \mathrm{~Hz}$. The gainbandwidth product will be given by:

$$
\mathrm{GB}=\left(\frac{1}{2 \pi T_{t}}\right) \frac{1}{1+\left(F / F_{\mathrm{o}}\right)^{n}}
$$

which also accounts for hole-trapping saturation at high excitation intensity. Despite the slow photocurrent relaxation time, the high gain values result in large gain-bandwidth products, implying that a significant photoresponse is expected in NW photodetectors even at high modulation frequencies. Figure $4 \mathrm{~b}$ shows the dynamic gain as a function of frequency in the range from 20 to $3000 \mathrm{~Hz}$, as obtained by modulating the light excitation source by means of a mechanical chopper and using a lock-in amplifier to measure the photocurrent. ${ }^{41}$ The gain values obtained are consistently high even in the submillisecond time domain: for instance, $G \sim 2 \times 10^{6}$ at $v=3 \mathrm{kHz}$, leading to $\mathrm{GB}=6 \mathrm{GHz}$. From eq 4 , one can infer a carrier transit time of $T_{\mathrm{t}} \sim 30 \mathrm{ps}$, corresponding to a carrier mobility of $\mu \sim 270 \mathrm{~cm}^{2} / \mathrm{V} \cdot \mathrm{s}$ for the $2 \mu \mathrm{m}$ electrode spacing at $V=5 \mathrm{~V}^{7}$ Because the dark current of these NW devices approaches $10 \mathrm{nA}$ at $V=5 \mathrm{~V}$
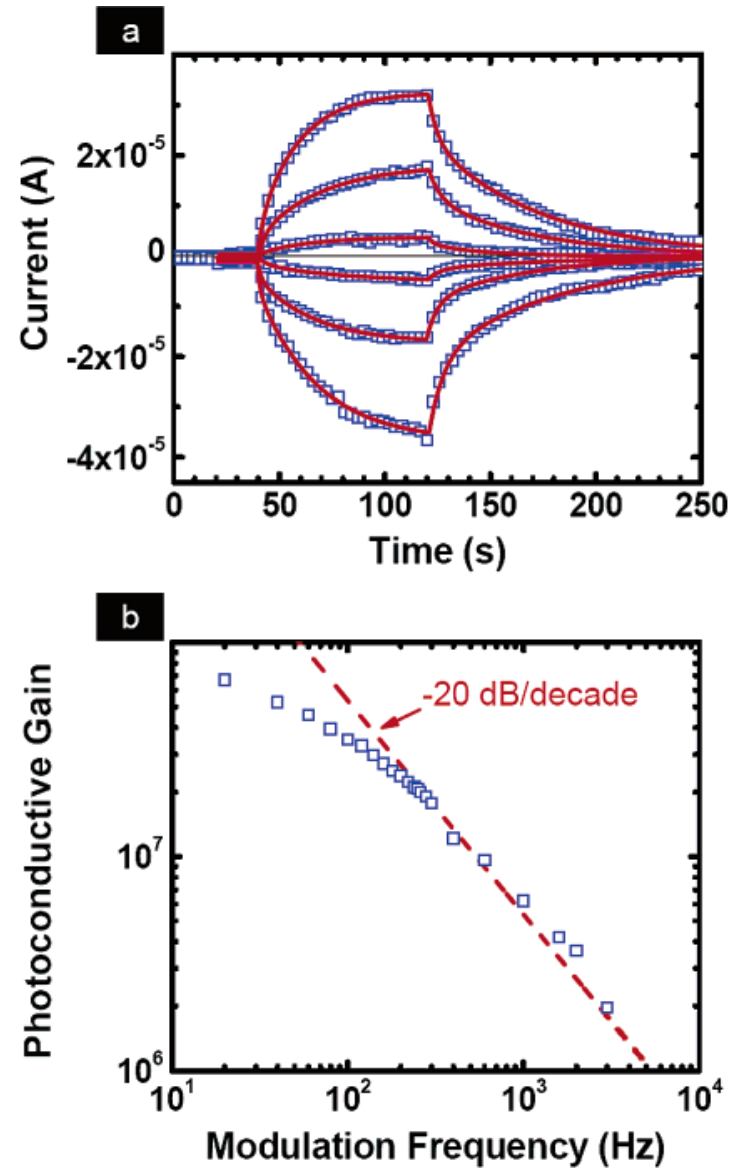

Figure 4. (a) Time-resolved photocurrent rise and decay as obtained by sudden application (at $40 \mathrm{~s}$ ) and removal (at $120 \mathrm{~s}$ ) of light illumination at different applied bias (from top to bottom, the curves were obtained with an applied external bias of $5,3,1,-1$, $-3,-5 \mathrm{~V}$, respectively); for each curve, the bias was applied after $20 \mathrm{~s}$; the excitation intensity was $I=4 \times 10^{-5} \mathrm{~W} / \mathrm{cm}^{2}$; the red lines are the best-fit to the data obtained with double-exponential rise and decay functions. (b) Frequency dependence of the photoconductive gain, with an excitation intensity of $1.6 \times 10^{-5}$ $\mathrm{W} / \mathrm{cm}^{2}(\lambda=390 \mathrm{~nm})$ and an external applied bias of $V=5 \mathrm{~V}$.

(Figure 1), we can then estimate the intrinsic carrier concentration to be as low as $n \sim 10^{13} \mathrm{~cm}^{-3}$, which indicates the high crystal quality of the $\mathrm{ZnO}$ NWs used in this study and explains the extremely high photoconductive gain observed. ${ }^{42}$

Because of oxygen adsorption and desorption at the NW surface, the carrier lifetime and hence the photocurrent intensity are strongly dependent on the ambient gas conditions. Figure 5a shows the photoconductivity spectra recorded in air and under vacuum, using the standard lock-in technique at a modulation frequency of $160 \mathrm{~Hz}$ and with an applied bias of $0.3 \mathrm{~V}$. In both cases, the photocurrent shows a sharp onset from 3.2 to $3.3 \mathrm{eV}$, corresponding to the $\mathrm{ZnO} \mathrm{NW}$ band gap. Above the band gap energy, the photoconductivity is almost constant (note the semilogarithmic scale) up to the deep UV spectral region, while no photoresponse has been detected in the range from 1.5 to $3.2 \mathrm{eV}$. The absence of photoconduction upon visible light illumination testifies to the low defect levels, ${ }^{17,18}$ such as oxygen vacancies in the $\mathrm{ZnO} \mathrm{NWs}$, which is supported by the low intrinsic electron concentration estimated above. The high spectral selectivity 

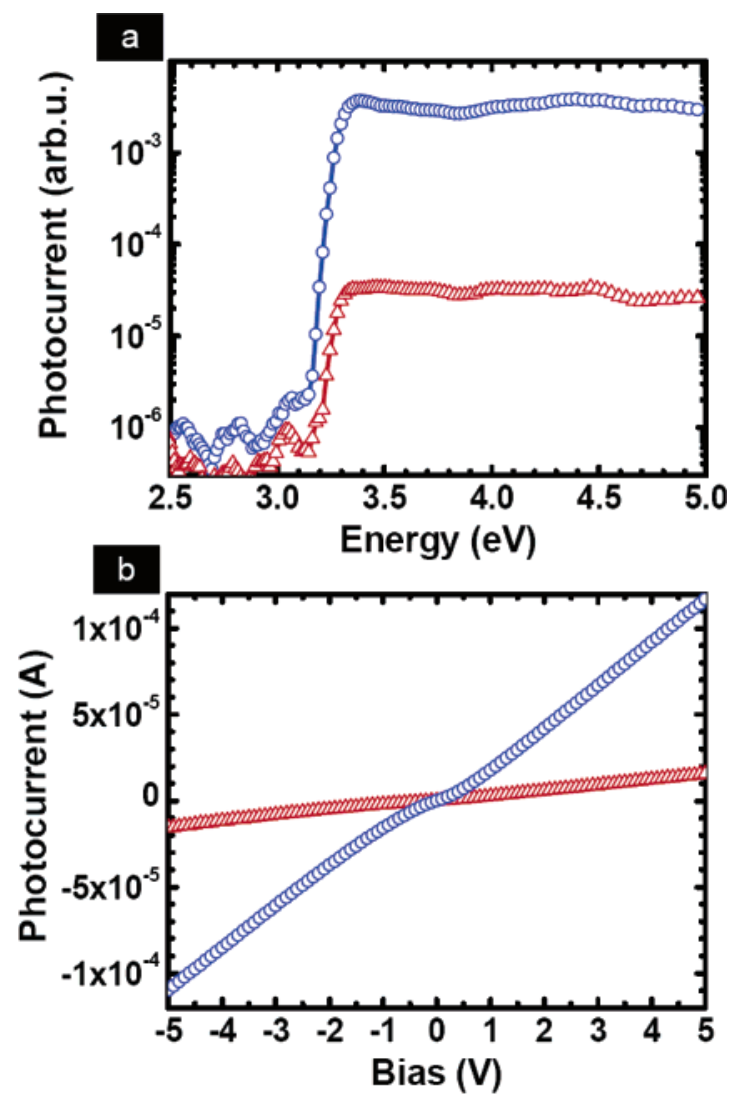

Figure 5. (a) Photocurrent spectra of an array of $\mathrm{ZnO}$ NWs obtained in air (red triangles) and in vacuum, at $P \sim 10^{-4}$ Torr (blue circles), with a modulation frequency of $160 \mathrm{~Hz}$ and an applied bias of $0.3 \mathrm{~V}$. (b) $I-V$ characteristics of a single $\mathrm{ZnO} \mathrm{NW}$ under illumination measured in air (red triangles) and in vacuum, at $P<$ $10^{-5}$ Torr (blue circles).

combined with high photosensitivity suggest the possibility of using ZnO NWs as "visible-blind" UV photodetectors for commercial, military, and space applications. ${ }^{43}$ Figure 5a also shows that the photoresponsivity of the $\mathrm{ZnO} \mathrm{NW}$ detector increases by about 2 orders of magnitude after keeping the sample under vacuum $\left(P<10^{-4}\right.$ Torr $)$ for approximately $20 \mathrm{~min}$. This increase in photocurrent is consistent with the increase of the electron lifetime due to a reduction in the oxygen readsorption rate in oxygen-deficient environments (Figure 2c), which is also supported by the spectral similarity of the photocurrent measured in air or in vacuum, indicating that the same physical processes are responsible for photoconduction. The increased trapping due to oxygen desorption is also manifested in $I-V$ measurements of single $\mathrm{NW}$ devices performed in vacuum $\left(P<10^{-4}\right.$ Torr $)$, where the photocurrent increases by a factor of more than 10 after pumping down for 5 min, as shown in Figure $5 b$. The fact that no change in the dark current has been observed in vacuum before illumination indicats that oxygen desorption is indeed facilitated by the photogeneration of holes that neutralize the oxygen ions at the surface.

We have also tested the high-frequency photoconductive response of $\mathrm{ZnO} \mathrm{NW}$ detectors by fast $(t<1 \mathrm{~ns})$ transient photocurrent measurements. ${ }^{44}$ The NW device was excited by ultrafast laser pulses (with pulse width of $t<150$ fs and repetition rate of $1 \mathrm{kHz}$ ), and the photocurrent decay was

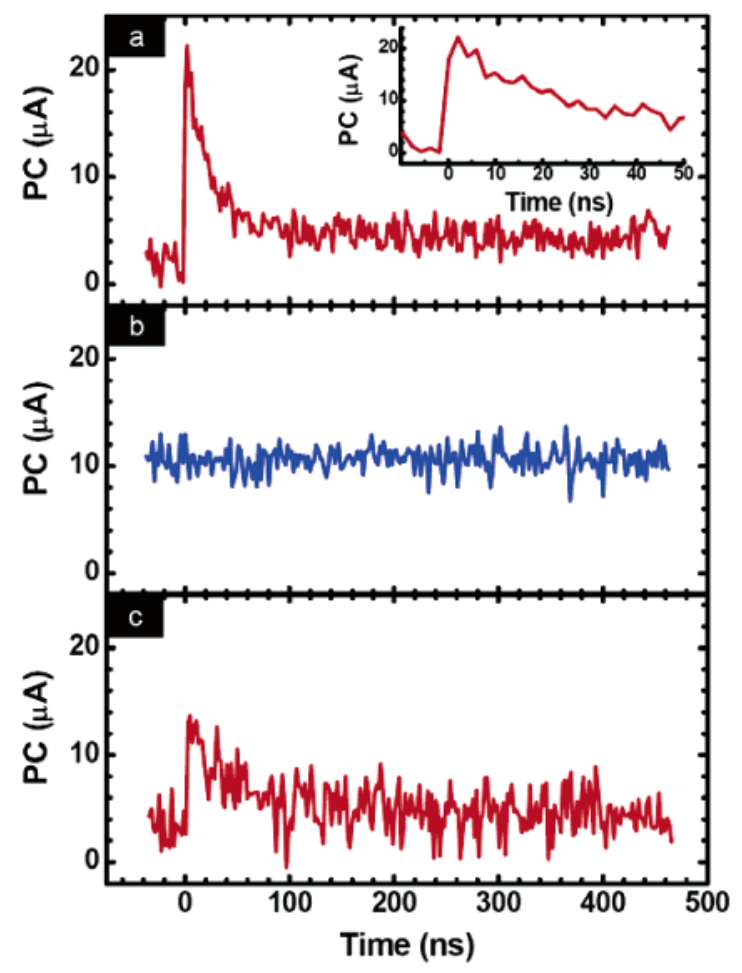

Figure 6. Fast transient photocurrent (PC) waveforms of $\mathrm{ZnO}$ NWs obtained (a) in air, (b) under vacuum $\left(P \sim 10^{-4}\right.$ Torr) and (c) reexposed to air for $1 \mathrm{~min}$ after vacuum. The excitation intensity was $I=0.3 \mu \mathrm{J} /$ pulse and the applied bias $V=1 \mathrm{~V}$. Inset of (a) shows the initial photocurrent decay in air, from which a characteristic time of $\sim 20$ ns can be inferred.

recorded using a fast boxcar integrator. ${ }^{45}$ To reveal the effect of oxygen adsorption and desorption on the photocurrent at fast time scales, we have performed the measurements while the sample was in standard ambient condition and in vacuum $\left(P<10^{-4}\right.$ Torr). Figure 6 shows the fast transient photocurrent decay waveforms of an array of $\mathrm{ZnO} N W s$ in different ambient conditions. Figure 6a shows the transient photocurrent measured in air, which displays a fast relaxation $(t \sim 20 \mathrm{~ns})$, consistent with our previous estimate for the GB product, followed by a long-lived photocurrent "tail" that persists over the whole time span investigated. ${ }^{46}$ For clarity, the data have been plotted on a different time scale in the inset of Figure 1a. The fast response in the nanoscond time scale demonstrates high-speed operation of NW photodetectors. Surprisingly, when the $\mathrm{ZnO}$ NW device was measured in vacuum, the fast component of the transient decay completely disappeared while the background current increased (Figure 6b). In vacuum and under intense illumination, oxygen molecules are discharged from the surface and thus the NWs are persistently photodoped, which results in higher dark current level and in the complete suppression of the photoresponse. Note that the ultrafast components of the photocarrier relaxation dynamics, such as the prompt electron-hole recombination and trapping, are not resolved in our measurements. ${ }^{47,48}$ The results in air and under vacuum indicate that the oxygen adsorption to and desorption from the NW surface occurs in very short time (ns), suggesting that the desorbed oxygen molecules in air remain in close proximity to the surface and can be promptly readsorbed. ${ }^{9}$ 
Figure $6 \mathrm{c}$ indicates that the oxygen readsorption to the surface and consequently the lowering of the dark current happen quickly after re-exposing the NWs to air; however, full recovery of the pristine conditions requires longer times, of the order of several minutes, due to the time required for oxygen molecules diffusion.

In summary, we have investigated the photoconductive properties of $\mathrm{ZnO} \mathrm{NW}$ for visible-blind UV photodetectors and fast optoelectronic applications. We have analyzed and quantified the photoconduction mechanism that leads to the substantial photoconductive gain measured $\left(G=2 \times 10^{8}\right)$, which, to the best of our knowledge, is among the highest reported gain for photodetectors. We have studied the photoconductivity of $\mathrm{ZnO}$ NWs using time-resolved measurements over a broad time range and in different ambient conditions (e.g., in air or under vacuum). We found that the photocarrier relaxation dynamics consists of a fast decay component in the nanosecond time range, which arises from the fast carrier thermalization and hole-trapping by surface states, followed by a "persistent" photocurrent, which decays within several seconds. We have also confirmed that, at all time spans investigated, the photosensitivity of $\mathrm{ZnO} \mathrm{NWs}$ and the photocarrier lifetime are considerably enhanced in oxygen deficient environments, consistent with the photoconductive mechanism involving oxygen adsorption and desorption at the NW surface. Surprisingly, this mechanism appears to be effective even at the shortest time scale investigated of $t<1 \mathrm{~ns}$. Despite the slow relaxation time, the extremely high internal gain of $\mathrm{ZnO}$ nanowire photodetectors results in gain-bandwidth products higher than GB $\sim 10 \mathrm{GHz}$. Furthermore, we have developed a physical model that illustrates the origin of the photoconductive gain in $\mathrm{ZnO} N W s$ and successfully predicts its functional dependence on excitation intensity and frequency. This model, broadly applicable to low-dimensional semiconductors with high density of surface trap states, demonstrates the uniqueness of NW as photodetectors with ultrahigh sensitivity and low power consumption for applications such as sensing, imaging, optical communications, and memory storage.

Acknowledgment. We acknowledge the support from the Office of Naval Research (ONR-Nanoelectronics), the National Science Foundation (ECS-0506902), and Sharp Labs of America. Spectral and fast transient photoconductivity measurements have been carried out at the Center for Polymers and Organic Solids at the University of California, Santa Barbara, and we are very grateful to Dr. Daniel Moses, Nelson Coates, and Prof. Alan Heeger for their help with the measurements and for useful discussions. We also thank Prof. Ed Yu at the University of California, San Diego, for allowing us to use the setup for $I-V$ measurements in vacuum. We acknowledge UCSD CALIT-2 and the staff of Nano3 Facility under CALIT-2 for supporting and maintaining a world-class nanofabrication environment.

\section{References}

(1) Karpina, V. A.; Lazorenko, V. I.; Lashkarev, C. V.; Dobrowolski, V. D.; Kopylova, L. I.; Baturin, V. A.; Pustovoytov, S. A.; Karpenko, A. J.; Eremin, S. A.; Lytvyn, P. M.; Ovsyannikov, V. P.; Mazurenko, E. A. Cryst. Res. Technol. 2004, 39, 980-992.
(2) Goldberger, J.; Sirbuly, D. J.; Law, M.; Yang, P. J. Phys. Chem. B 2005, 109, 9-14.

(3) Ng, H. T.; Han, J.; Yamada, T.; Nguyen, P.; Chen, Y. P.; Meyyappan, M. Nano Lett. 2004, 4, 1247-1252.

(4) Pauzauskie, P. J.; Yang, P. Mater. Today 2006, 9, 36-45.

(5) Zou, B. S.; Liu, R.; Wang, F.; Pan, A.; Cao, L.; Wang, Z. L. J. Phys. Chem. B 2006, 110, 12865-12873.

(6) Wang, Z. L. Annu. Rev. Phys. Chem. 2004, 55, 159-196.

(7) Özgür, Ü.; Alivov, Y. I.; Liu, C.; Teke, A.; Reshchikov, M. A.; Doan, S.; Avrutin, V.; Cho, S.-J.; Morkoç, H. J. Appl. Phys. 2005, 98, 041301.

(8) Kind, H.; Yan, H.; Messer, B.; Law, M.; Yang, P. Adv. Mater. (Weinheim, Germany) 2002, 14, 158-160.

(9) Law, J. B. K.; Thong, J. T. L. Appl. Phys. Lett. 2006, 88133114.

(10) Luo, L.; Zhang, Y. F.; Mao, S. S.; Lin, L. W. Sens. Actuators, A 2006, 127, 201-206.

(11) Suehiro, J.; Nakagawa, N.; Hidaka, S.; Ueda, M.; Imasaka, K.; Higashihata, M.; Okada, T.; Hara, M. Nanotechnology 2006, 17, 2567-2573.

(12) Konenkamp, R.; Word, R. C.; Schlegel, C. Appl. Phys. Lett. 2004, 85, 6004-6006.

(13) Kumar, S.; Gupta, V.; Sreenivas, K. Nanotechnology 2005, 16, $1167-$ 1171

(14) Heo, Y. W.; Tien, L. C.; Norton, D. P.; Kang, B. S.; Ren, F.; Gila, B. P.; Pearton, S. J. Appl. Phys. Lett. 2004, 85, 2002-2004.

(15) Hsu, C.-L.; Chang, S.-J.; Lin, Y.-R.; Li, P.-C.; Lin, T.-S.; Tsai, S.Y.; Lu, T.-H.; Chen, I. C. Chem. Phys. Lett. 2005, 416, 75-78.

(16) Heo, Y. W.; Kang, B. S.; Tien, L. C.; Norton, D. P.; Ren, F.; La Roche, J. R.; Pearton, S. J. Appl. Phys. A: Mater. Sci. Process. 2005, 80, 497-499.

(17) Keem, K.; Kim, H.; Kim, G.-T.; Lee, J. S.; Min, B.; Cho, K.; Sung, M.-Y.; Kim, S. Appl. Phys. Lett. 2004, 84, 4376-4378.

(18) Fan, Z. Y.; Chang, P. C.; Lu, J. G.; Walter, E. C.; Penner, R. M.; Lin, C. H.; Lee, H. P. Appl. Phys. Lett. 2004, 85, 6128-6130.

(19) Soci, C.; Bao, X. Y.; Zhang, A.; Liu, J.; Wang, D. J. Nanosci. Nanotechnol. 2007, to be submitted.

(20) Bube, R. H. Photoelectronic Properties of Semiconductors; Cambridge University Press: Cambridge, 1992.

(21) Rose, A. Concepts in Photoconductivity and Allied Problems; Interscience Publishers: New York, 1963.

(22) Jie, J. S.; Zhang, W. J.; Jiang, Y.; Meng, X. M.; Li, Y. Q.; Lee, S. T. Nano Lett. 2006, 6, 1887-1892.

(23) Xiang, B.; Wang, P.; Zhang, X.; Dayeh, S. A.; Aplin, D. P. R.; Soci, C.; Yu, D.; Wang, D. Nano Lett. 2006, 7, 323-328.

(24) $I-V$ measurements in the dark and under illumination were obtained by applying an external bias to the NW and recorded using a lownoise current preamplifier (Ithaco 1211) in conjunction with a 100 $\mathrm{kS} / \mathrm{s}, 16$ bit digital acquisition board (National Intruments PCI$6030 \mathrm{E}$ ). The time delay between acquisitions was set at $t=100 \mathrm{~ms}$. A $\mathrm{Hg}$ arc discharge lamp was used as the excitation source, and monochromatric UV illumination was obtained by a dielectric bandpass filter centered at $390 \mathrm{~nm}( \pm 50 \mathrm{~nm})$ placed in front of the lamp. The optical power impinging on the nanowire photodetectors was varied by means of neutral density filters. The illumination intensity was determined by a thermopile detector (SpectraPhysics 407A). Measurements under vacuum were obtained by placing the samples in the vacuum chamber of a "cold" probe station (MMR Technologies, Inc.) at a pressure of $P<10^{-4}$ Torr.

(25) Takahashi, Y.; Kanamori, M.; Kondoh, A.; Minoura, H.; Ohya, Y Jpn. J. Appl. Phys., Part 1 1994, 33, 6611-6615.

(26) Li, Q. H.; Gao, T.; Wang, Y. G.; Wang, T. H. Appl. Phys. Lett. 2005 , $86,123117 / 1-123117 / 3$.

(27) Li, Q. H.; Wan, Q.; Liang, Y. X.; Wang, T. H. Appl. Phys. Lett. 2004, 84, 4556-4558.

(28) Li, Q. H.; Liang, Y. X.; Wan, Q.; Wang, T. H. Appl. Phys. Lett. 2004, 85, 6389-6391.

(29) Mehta, R. R.; Sharma, B. S. J. Appl. Phys. 1973, 44, 325-328.

(30) Matsuo, N.; Ohno, H.; Hasegawa, H. Jpn. J. Appl. Phys., Part 2 1984, 23, L299-L301

(31) Vilcot, J. P.; Vaterkowski, J. L.; Decoster, D.; Constant, M. Electron. Lett. 1984, 20, 86-88.

(32) Katz, O.; Garber, V.; Meyler, B.; Bahir, G.; Salzman, J. Appl. Phys. Lett. 2001, 79, 1417-1419.

(33) Futako, W.; Kamiya, T.; Fortmann, C. M.; Shimizu, I. J. Non-Cryst. Solids 1998, 230, 220-224.

(34) Dohler, G. IEEE J. Quantum Electron. 1986, 22, 1682-1695.

(35) Kurtz, S. R.; Biefeld, R. M.; Dawson, L. R.; Fritz, I. J.; Zipperian, T. E. Appl. Phys. Lett. 1988, 53, 1961-1963. 
(36) Bube, R. H. Photoconductivity of Solids; Wiley: New York, 1960; p 461.

(37) To estimate $P$, we have considered a NW surface area between the contacts of $A=1.2 \times 10^{-8} \mathrm{~cm}^{2}$ and assumed $85 \%$ absorption of the incident power based on $\sim 10 \%$ surface reflection and $\sim 95 \%$ absorption in the $\sim 300 \mathrm{~nm}$ diameter $\mathrm{NW}$ at $3.5 \mathrm{eV}$ (see Zhang, Z. H.; Qi, X. Y.; Jian, J. K.; Duan, X. F. Micron 2006, 37, 229233).

(38) Hayden, O.; Agarwal, R.; Lieber, C. M. Nat. Mater. 2006, 5, 352356.

(39) Yang, C.; Barrelet, C. J.; Capasso, F.; Lieber, C. M. Nano Lett. 2006, 6, 2929-2934.

(40) Soci, C.; Moses, D.; Xu, Q. H.; Heeger, A. J. Phys. Rev. B 2005, $72,245204$.

(41) The frequency and spectral dependence of the photocurrent were obtained using a conventional modulation technique, where the light emitted by a Xe arc discharge lamp, modulated by a mechanical chopper (EG\&G-PAR 197, frequency range up to $3000 \mathrm{~Hz}$ ), was used as the excitation source, in conjunction with a monochromator and a lock-in amplifier (Ithaco 3921 or Stanford Research SR520) for measuring the photocurrent. For determining the photocurrent spectra, the raw data were calibrated against the excitation intensity determined by means of a calibrated Si photodiode (Newport 818UV).

(42) The experimental value of the carrier lifetime $\left(T_{1}=33 \mathrm{~s}\right)$ and the estimated transit time $\left(T_{\mathrm{t}}=30 \mathrm{ps}\right)$ lead to an upper limit for the DC gain at low excitation level of $G \sim 10^{12}$ (eq 3 ), somewhat larger than the values derived in Figure $3 \mathrm{~b}$ (at a bandwidth of $B=10 \mathrm{~Hz}$ ) and Figure $4 \mathrm{~b}$ (with a light intensity of $I=1.6 \times 10^{-5} \mathrm{~W} / \mathrm{cm}^{2}$ ), but consistent with the extrapolation at zero frequency of the $-20 \mathrm{~dB} /$ decade line shown in Figure 4b. The deviation of the experimental data from the expected gain values at low modulation frequencies may be due to the presence of multiple time constants, which would result in multiple poles and zeros of the transfer function $G(v)$.

(43) Razeghi, M.; Rogalski, A. J. Appl. Phys. 1996, 79, 7433-7473.

(44) Auston, D. H. IEEE J. Quantum Electron. 1983, 19, 639-648.

(45) Fast transient photocurrent measurements were obtained by the Auston-switch technique, where $\mathrm{ZnO}$ nanowires were mechanically transferred onto a glass substrate and incorporated into a gold microstrip line with a gap between the electrodes of $d=4 \mu \mathrm{m}$. The optical excitation was generated by the third harmonic $(\lambda=267 \mathrm{~nm})$ of a Ti:sapphire laser system (SpectraPhysics) with pulse duration of $<150 \mathrm{fs}$, and the transient photocurrent was measured using a fast boxcar integrating system (EG\&G-PAR 4400); the overall temporal resolution of the measuring setup was less than $1 \mathrm{~ns}$. Measurements in vacuum were performed in the vacuum chamber of a homemade cryostat at a pressure of $<10^{-4}$ Torr.

(46) It is noteworthy that, due to the extremely high photon flux required to obtain a satisfactory signal-to-noise ratio in these measurements, the gain values that would correspond to the photocurrent transients in Figure 6a are well below unity, as expected from the gain saturation at such excitation intensities $(I=0.3 \mu \mathrm{J} / \mathrm{pulse})$.

(47) Cooke, D.; Wu, Z.; Mei, X.; Liu, J.; Ruda, H. E.; Kavanagh, K. L.; Hegmann, F. A. In Transient photoconductivity of GaAs and AlGaAs nanowires; American Physical Society, March Meeting 2004, Palais des Congres de Montreal, Montreal, Quebec, Canada, March 2226, 2004, 2004; Palais des Congres de Montreal: Montreal, Quebec, Canada, 2004.

(48) Baxter, J. B.; Schmuttenmaer, C. A. J. Phys. Chem. B 2006, 110, 25229-25239.

NL070111X 